\title{
Stereopsis impairment in apparently moving random dot patterns
}

\author{
I. HADANI and N. VARDI \\ Israel Institute of Technology, Haifa, Israel
}

\begin{abstract}
A moving random-dot pattern was stereoscopically partitioned into a static vertical cyclopean depth grating. The dots were horizontally displaced across the grating in repeated uniform jumps. Stereopsis and movement perception were examined at stimulus angular velocities in the range of $0.1^{\circ}-44^{\circ} / \mathrm{sec}$. At $1^{\circ}-5.5^{\circ} / \mathrm{sec}$ the depth perception of the grating was strongly impaired: that is, depth judgment for suprathreshold disparities was poor and the stereo-acuity threshold was elevated. The effect of varying the cyclopean spatial frequencies (CSFs) between 0.3 and $4.2 \mathrm{cpd}$ was also examined. Although there was no strong dependence on CSF, the impairment was more pronounced at CSFs greater than $1.3 \mathrm{cpd}$. When smooth tracking eye movements were absent, that is, during fixation or saccadic scan, stereoscopic depth was not impaired. Eye movement measurements indicated that this phenomenon is associated with optokinetic nystagmus (OKN). It is suggested that the impairment in resolving depth in this particular spatiotemporal configuration actually reflects a temporal limit of the stereoscopic system, which is manifested during OKN.
\end{abstract}

Random-dot patterns are becoming increasingly popular as psychophysical visual stimuli. These relatively simple unstructured stimuli enable us to stimulate, in isolation, specific perceptual mechanisms. This is especially important when many interacting mechanisms operate in parallel at different hierarchical levels, as is the case in visual processing. In binocular vision studies, randomdot stimuli were extensively employed to stimulate the cyclopean retina without the contaminating effects of monocular cues for depth (Julesz, 1971). Similarly, in many studies of motion perception, these random-dot patterns were found to be effective for examining early processing of motion (Nakayama, Silverman, MacLeod, \& Mulligan, 1985). Relatively few studies have employed random-dot techniques for simultaneous stimulation of stereopsis and (monocular) movement perception in order to explore possible interactions between these two mechanisms (Hadani, 1984a; Julesz, 1971, pp. 82-85; Lee, 1970). Dynamic random-dot stereograms (RDSs) are particularly advantageous for this type of study. Changes in the disparity and movement fields are correlated in the case of real dynamic 3-D objects (Gibson, 1966; Lee, 1970); here, in contrast, the field parameters can be manipulated independently. Therefore, everyday-like as well as highly artificial dynamic 3-D objects can be perceptually simulated and explored.

This project was supported in part by the National Council for Research and Development-the Israeli Ministry of Science and Development (Grant 2519), and in part by the Starr Fund for Ophthalmological Research (Grant 130-099). The authors are indebted to M. Gur, Y. Norman, and P. Meretsky for reviewing the manuscript and to Z. Smolinsky for technical assistance. Address correspondence to I. Hadani, who is now at the Institute for Perception Research, P.O. Box 513, 5600 MB Eindhoven, The Netherlands.
Our original goal was to address questions related to the interaction between stereopsis and motion mechanisms. We exploited the advantages offered by dynamic RDS techniques and devised a stereoscopic computercontrolled CRT display in which the random dots coherently moved across a static stereograting in repeated uniform jumps. The cyclopean spatial frequency (CSF) and the dots' virtual angular velocity (AV) were independently manipulated. In preliminary observations we found that stereopsis was impaired at relatively low AVs of the dots $\left(1^{\circ}-3 \% / \mathrm{sec}\right)$, while the coherent motion of the dots was preserved. A similar phenomenon was noticed by Adler and Grüsser (1982), in relation to the sigma-movement phenomenon, but these authors concentrated on the stimulus conditions under which stereopsis was preserved. In order to understand the nature of the stereopsis impairment, we investigated the effect by changing CSF and AV. These investigations indicated that the impairment was associated with optokinetic nystagmus (OKN), which was evoked by the coherently moving dots. In this work we therefore concentrated on understanding the role of eyemovement behavior in the stereopsis impairment. The results are analyzed in light of other relevant reports and an attempt is also made toward understanding the perceptual and neural sources of the phenomenon.

The present work is related to two relatively new apparent-motion phenomena that are basically monocular. The first is the alpha-stripes effect, which appears when the observer's eyes are fixated on a repeatedly displaced random-dot pattern, or when the eyes are smoothly tracking (by pursuit or OKN) a stationary but stroboscopically illuminated random-dot pattern (Adler, Bock, \& Grüsser, 1981; Adler \& Grüsser, 1979; Koenderink \& Van Doorn, 1980). The appearance of the stationary stripes superimposed on the moving pattern involves two 
processing channels operating simultaneously on the same dynamic visual input. These channels, the pattern detection and movement detection systems, differ in their spatiotemporal sensitivities. The alpha stripes presumably result from the long time constant of the pattern detection system $(\sim 0.5 \mathrm{sec})$, which summates the luminosity of successive, identical but incrementally displaced, frames.

The second effect is "pursuit-locked apparent motion," which is a modern version of the long-standing retinalpainting phenomenon (Farrell, Putnam, \& Shepard, 1984; Morgan, 1981). It is obtained when a luminous element (a dot or vertical bar) is presented in repeated discrete lateral jumps but persists in each position for $\tau<$ 100 msec. When the apparently moving stimulus is smoothly tracked, the persistent part of the stimulus is painted on the retina and appears as a 2-D extension of the apparently moving element (Hadani, 1984b).

Our stereoscopic display met the conditions for both the alpha-stripes and retinal-painting effects, because it involved repeated apparent motion of elements that persisted for short durations ( $<60 \mathrm{msec}$ ) on the phosphor screen. The alpha stripes appeared when the subjects fixated or scanned the stationary stereograting, and the retinal-painting effect prevailed when OKN was elicited by the coherently moving field. The retinal-painting effect was expressed by the appearance of trailing cometlike tails attached to the moving dots. In this study we did not investigate these two monocular effects, but used them qualitatively to probe the observers' eye-movement behavior.

\section{METHOD}

\section{Apparatus}

Stereoscopic random-dot arrays composed of 1,000 dots each were presented in $x y$ mode on two Tektronix oscilloscopes with P1 phosphor screens (10 msec to below $10 \%$ of maximum brightness). The oscilloscope screens, positioned at right angles to each other, were superimposed by a $0.5 / 0.5$ beam splitter. Crossed Polaroid filters on the screens and on the subjects' eyeglasses separated fields to the left and right eyes. The dynamic random-dot arrays were generated on-line and were refreshed by a PDP 11/55 computer, equipped with an LPA interface. The presentation of a single dot of a stereopair ( $20 \mu \mathrm{sec}$ in duration) on a single scope was immediately followed by the presentation of its binocularly corresponding dot on the second scope. Unless otherwise specified, the frame rate30 frames/sec-was kept constant throughout all the experiments.

\section{Display and Procedure}

The display subtended $3.1^{\circ} \times 2.6^{\circ}$ from the $110-\mathrm{cm}$ viewing distance. Unless otherwise stated, average dot density was $124 \mathrm{dots} / \mathrm{deg}^{2}$; individual dot size was $3 \mathrm{arcmin}$, and dot luminance was about $2 \mathrm{~cd} / \mathrm{m}^{2}$ against a dark background.

The basic display is presented in Figure 1. The random-dot pattern was partitioned into vertical cyclopean static stripes of equal width alternating in depth (depth grating modulation). In addition, the random-dot pattern was unidirectionally shifted in discrete horizontal jumps. CSF, AV, and disparity were altered by the computer program. CSF ranged from 0.3 to $4.2 \mathrm{cpd}$ and AV in the basic display dimensions ranged from $0 \% \mathrm{sec}$ to $4.4 \% \mathrm{sec}$. Higher AVs, up to $44 \% \mathrm{sec}$, were obtained by horizontal expansion of the display.
(A) STATIC DEPTH. GRATING

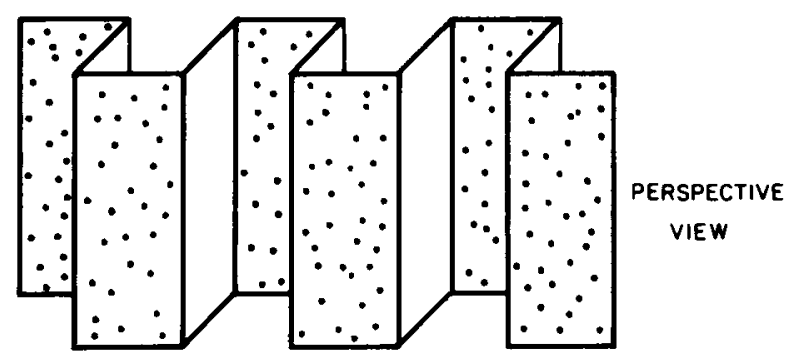

(a)

RANOOM DOTS IN COHERENT APPARENT MOTION

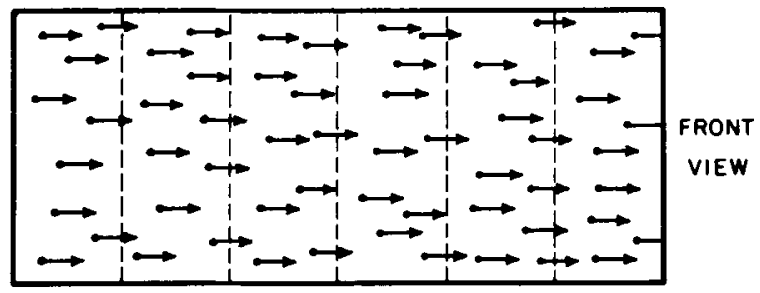

Figure 1. (A) Schematic illustration of the perceived form of the stereoscopic display. A thousand dots were randomly distributed over a field of $2.6^{\circ} \times 3.1^{\circ}$ and were divided into vertical static stripes alternating in depth. (B) Front view of the display. The arrows designate the horizontal uniform discrete displacements.

In a static condition, that is, when the dots did not move, distinct stripes with sharp stereoscopic edges were observed up to $4.7 \mathrm{cpd}$. Above this spatial frequency, up to $165 \mathrm{cpd}$ (which was the highest possible CSF), the dots appeared to create homogeneous planes at two depth levels. In the dynamic case the stereograting remained in a constant position with respect to the CRT screen frame despite the dots' repeated lateral movement. Therefore, when a given dot crossed a stereoscopic edge, say from a farther to a closer stripe, it acquired in addition to its movement step $(\Delta x)$ a nonzero disparity increment $(\delta x)$, and its total displacement then was $\Delta x+\delta x$; thereafter, it retained its $\Delta x$ jumps. When it moved to the next neighboring stripe it acquired a disparity decrement of $\delta x$, and its displacement while crossing was $\Delta x-\delta x$. This was obtained by an appropriate manipulation of the dots' $x$-coordinate values on one scope only (scope A). On the other scope (B), all the dots had constant $\Delta x$ jumps. As a result of the disparity modulation of scope $A$ dots, monocular vertical dark contours appeared for large values of $\delta x$. Also, the monocular movement patterns of the dots were not seen as purely homogeneous, due to the acceleration and deceleration of the edge-crossing dots. However, these lateral movement irregularities completely disappeared in stereoscopic observation and the edge-crossing dots were seen to move in depth.

We also note that for the small values of disparity utilized in the present experiments ( $\delta x<2$ arcmin), the vertical dark bars were not even visible monocularly and presumably did not play a major role in stereopsis impairment (see Discussion).

In all the formal psychophysical measurements, a two-alternative forced-choice paradigm was employed. The subject's task was to determine whether the odd stripes were closer or farther than the even stripes. In all experiments, excluding the stereoacuity experiment, $\mathrm{AV}$ was the independent variable, having six values in a logarithmic scale. Disparity magnitude (1.8 arcmin) and CSF were kept constant within a session. In subsequent sessions the CSFs were randomly selected from a preordained listing.

For the stereoacuity experiment, AV and CSF were kept constant within a session and the AV was randomly selected between 
sessions. In a typical session, each of six values of the relevant independent variable was selected 10 times at random. Also, in the early experiments (Figure 2), the dots moved in only one direction. In later experiments, right and left direction were randomly employed. This directional change had no significant effect on the data. Unless otherwise specified, a single exposure lasted $6 \mathrm{sec}$ or until the subject responded. For each threshold determination, at least 200 responses were collected and the resultant psychometric function was fitted with the aid of a Probit procedure (SAS package). Threshold criterion was $75 \%$ correct.

\section{Recording of Eye Movements}

Horizontal movements of the right eye were measured with an IR corneal reflection Eye Track Monitor (Biometrics Model 200) and recorded on a Bryans Southern Model 10-500 UV recorder. The subject signaled the occurrence of stereopsis by operating a microswitch connected to the marker channel of the recorder.

\section{Observers}

Three observers with normal or corrected-to-normal vision participated in the study. All had good stereopsis, that is, a disparity threshold of 9.7" as tested by a Bausch \& Lomb orthorater. Two of the subjects were highly trained in visual psychophysics, whereas the third (T.C.) was a naive subject. Data were collected only after improvement in performance due to learning was negligible.

\section{RESULTS}

Figure 2 shows the effect of AV on stereoscopic depth perception of vertical stripes for 3 subjects at five different spatial frequencies. At AVs of up to $0.37^{\circ} / \mathrm{sec}$, stereopsis was easily obtained. The percept was that of a slow unidirectional flow of the dots across the stereograting. At higher AVs $\left(1^{\circ}-3^{\circ} / \mathrm{sec}\right)$, the moving dots acquired comet-like tails and the depth percept of the grating was impaired. A unidirectional flow of the pattern in one depth plane was the dominant percept. The effect of increasing spatial frequency, although not very pronounced, tended to reduce performance. Two main individual differences are shown in the results: (1) the performance of Subject Z.S. was much better than that of the other 2 subjects, and (2) whereas Subjects T.C. and Z.S. showed monotonic decreases in performance with increasing AVs, Subject N.V. showed a recovery or improvement in performance at $3 \%$ sec.

These differences notwithstanding, stereopsis was impaired for all subjects in the range of $1^{\circ}-3^{\circ} / \mathrm{sec}$. Informal observations with untrained subjects having normal stereopsis showed a complete loss of depth perception even at lower AVs and/or CSFs.

\section{The Effect of Stimulus AV on Stereoacuity}

For each of five values of $\mathrm{AV}$ in the range $0 \% \mathrm{sec}$ (static conditions) to $4.4 \% \mathrm{sec}$, a stereo threshold was determined from a psychometric function of response rates versus disparity. The results are presented in Figure 3 for Subjects N.V. and Z.S. There was a clear increase in threshold values for both subjects as AV was increased. In agreement with the previous results, a slight threshold decrease was shown by Subject N.V. at $3 \%$ sec.
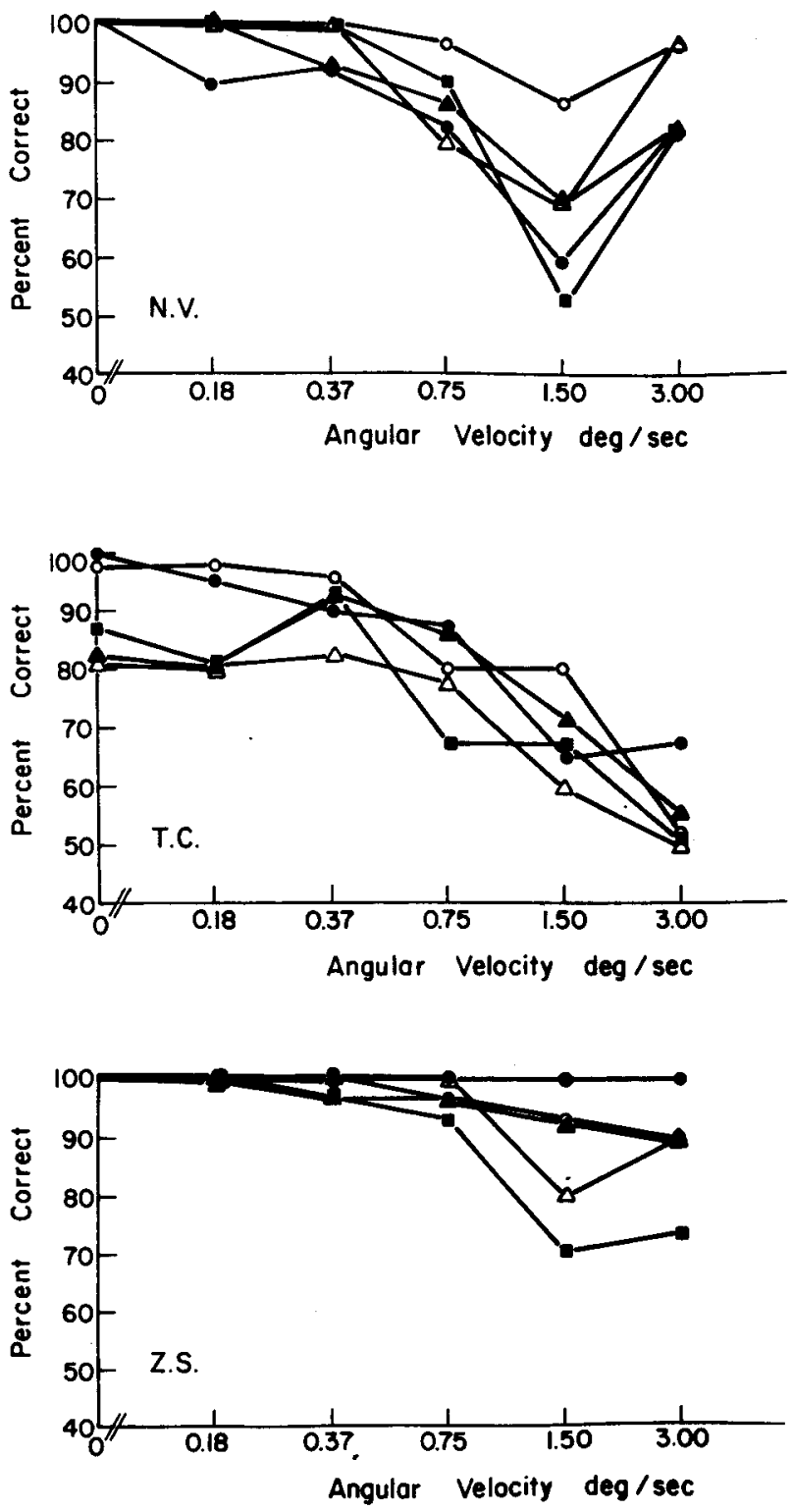

Figure 2. Effect of velocity on stereopsis for 3 subjects. Each point represents 30 trials. Different symbols designate different cyclopean spatial frequencies as follows: open circles-0.3 cpd; closed circles1.3 cpd; closed squares -2.3 cpd; closed triangles -3.2 cpd; open triangles -4.2 cpd.

\section{Stereopsis at Higher Stimulus AVs}

Because of the improvement in Subject N.V.'s performance at the higher AVs, we suspected that the curve might change direction for the other subjects at higher stimulus AVs. Therefore, we replicated the first (Figure 2) experiment at three overlapping intervals ranging from $0.275 \% \mathrm{sec}$ to $44^{\circ} / \mathrm{sec}$. The extension of AV was achieved by expanding the display horizontally. This resulted in a field-width increase and a dot-density decrease. Nevertheless, the impairment, centered at about $3 \% \mathrm{sec}$, was clearly evident (Figure 4 ). These results show 


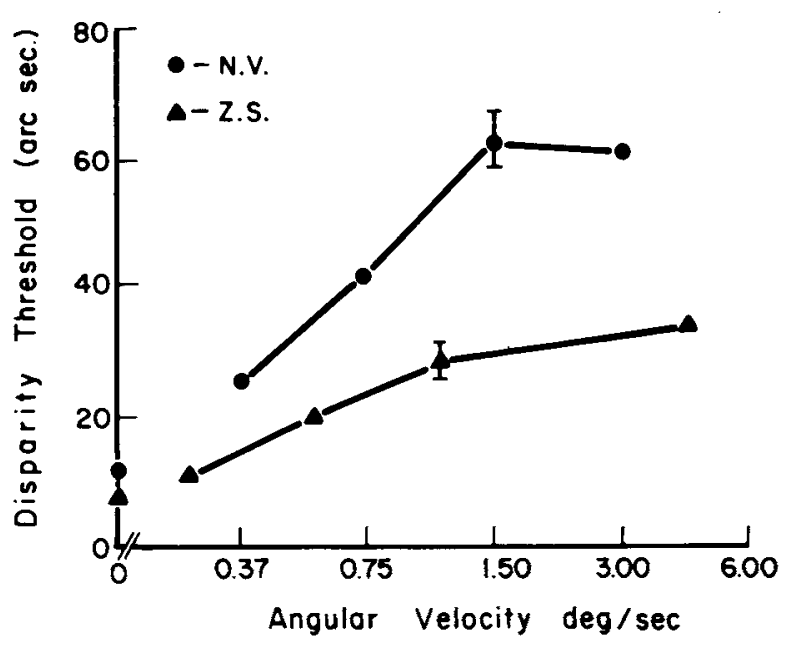

Figure 3. The effect of stimulus angular velocity on disparity threshold for Subjects N.V. and Z.S. For Subject N.V., cyclopean spatial frequency $(\mathrm{CSF})=0.66 \mathrm{cpd}$; for Subject Z.S., CSF $=$ 3.2 cpd. Standard errors were calculated for two representative data points.

that as the dots' virtual velocity is increased above $3 \% \mathrm{sec}$, there is a gradual improvement in performance that levels off at $11 \% / \mathrm{sec}$, where it reaches $100 \%$ correct responses. Although the effects of field size and density were not investigated, it is unlikely that the limited AV range of impairment in stereopsis demonstrated in these experiments would have drastically changed had these parameters been controlled.

The subjects reported on a striking relationship between the percept of the moving dots and that of the perceived stereopattern at different dots' AVs. At low AVs $(<1 \% \mathrm{sec})$, they perceived a slow dot flow and stereopsis was easily obtained. At moderate AVs $\left(1^{\circ}-5.5^{\circ} / \mathrm{sec}\right)$, the dots acquired comet-like trailing tails due to the CRT phosphor persistence (Hadani, 1984b), and stereopsis was very difficult to obtain. At high AVs $(>12 \% \mathrm{sec})$, the alpha-stripes percept (Adler et al., 1981; Adler \& Grüsser, 1979; Koenderink \& Van Doorn, 1980; see also Discussion) was dominant and stereopsis was again easily obtained. Between the moderate and high AVs $\left(5.5^{\circ}-12^{\circ} / \mathrm{sec}\right)$, there was a region that gave rise to bistable percepts. In this region, either the comet-tails or the alpha-stripes percept was obtained. Whenever the comet tails appeared, stereopsis ceased. Whenever the alphastripes percept appeared, the vertical stereo stripes reappeared. In examining this phenomenon we discovered that the comet-tails percept was observed only during $\mathrm{OKN}$ and the alpha-stripes percept only during fixation. These observations led us to suspect that OKN plays a major role in the stereopsis impairment phenomenon.

\section{The Dependence of Stereopsis Impairment on OKN}

We confirmed the hypothesized association between the impairment in stereopsis and OKN directly, by eyemovement measurements, and indirectly, by responses to stimuli that did not evoke OKN. The eye movements of one subject (N.V.) were measured at few representative AVs, while she was observing the stereodisplay. At low AVs when stereopsis was not impaired, no characteristic OKN could be found (Figure 5A). The mean number of occasional OKN sweeps was increased with AV. This was true for AVs up to $3 \% \mathrm{sec}$, where stereopsis ceased and OKN dominated the eye-movement records (not shown). The correlation could best be demonstrated at $4.8 \% \mathrm{sec}$ (Figure 5B), where spontaneous transitions from saccadic scan to OKN periods were accompanied by transitions from stereopsis to nonstereopsis periods.

OKN was eliminated during task performance by using brief exposure durations or by utilizing oscillatory motion. Since it is known that the latency of OKN is $125-180 \mathrm{msec}$ (Westheimer, 1954), the vertical stereo stripes were presented for only $100 \mathrm{msec}$. The results for 2 subjects are presented in Figure 6. Unlike in the first experiment, stereopsis was not significantly degraded up to $4.4 \% \mathrm{sec}$. The overall poorer performance probably resulted from the inherent difficulty in performing this task at short exposure durations. In an additional test, the unidirectional-flow motion of the dots was replaced by a to-and-fro motion with a rate of 30 or 15 frames per second. The to-and-fro displacement magnitudes varied from 0.5 arcmin to 8 arcmin, which were equivalent to $0.275 \% / \mathrm{sec}$ and $4.4 \% \mathrm{sec}$, respectively, had the discrete jumps been unidirectional. Spatial frequency was also varied from 0.33 to $4.3 \mathrm{cpd}$. No impairment in stereopsis was observed under any AV-CSF combination tested. The vertical stripes in depth were clearly seen by all subjects.

\section{DISCUSSION}

The present study demonstrates an impairment in stereopsis under highly artificial dynamic stimulus conditions that allow differential stimulation of the motion and stereoscopic mechanisms. We had no a priori reason to suspect that an elementary visual function such as stereopsis would be impaired at relatively low AVs of the dots and at low stereograting spatial frequencies. This is especially true in view of the finding that lateral motion up to $2 \% / \mathrm{sec}$ does not affect stereoacuity (Westheimer \& McKee, 1978). In our experiment, stereoacuity was already impaired at $0.2 \% \mathrm{sec}$ (Figure 3). Two important differences exist between Westheimer and McKee's study and ours, which may account for the disparate results. First, Westheimer and McKee utilized 200-msec exposure durations, whereas we used prolonged presentation. These prolonged exposure durations of the coherently moving field triggered $O K N$, which was not probable at the short durations in Westheimer and McKee's study. Second, Westheimer and McKee displaced the entire 3-D stimulus, whereas in our study the 3-D structure remained static and only the dots moved. We will argue that the impairment in stereopsis is due to both OKN and the stationariness of the stereograting. 


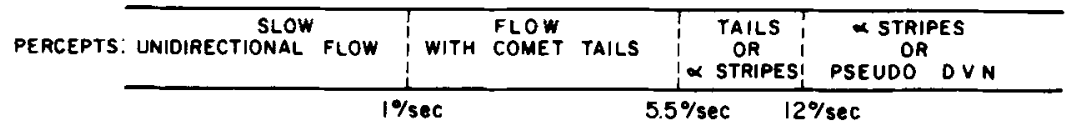

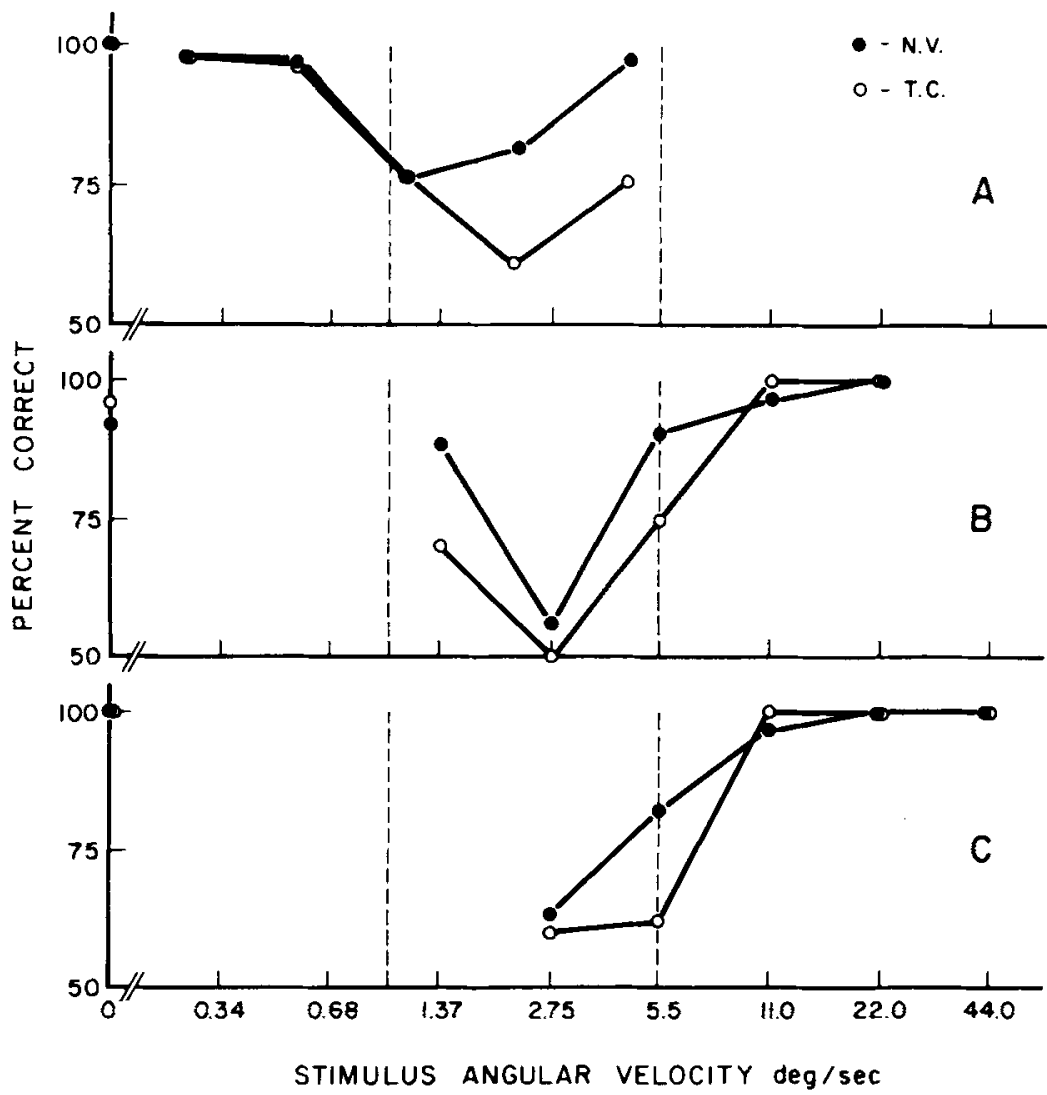

Figure 4. The effect of an extended range of angular velocities on stereopsis. Results are for 2 subjects. (A) Basic display size $=2.60^{\circ} \times 3.10^{\circ}$, dot density $=124 \mathrm{dots} / \mathrm{deg}^{2}$; cyclopean spatial frequency (CSF) $=2.3 \mathrm{cpd}$. (B) Horizontal axis expanded 5 times: display size $=2.6^{\circ} \times 6.2^{\circ}$; dot density $=25 \mathrm{dots} / \mathrm{deg}^{2} ; \mathrm{CSF}=1.8 \mathrm{cpd}$. (C) Horizontal axis expanded 10 times: display size $=2.6^{\circ} \times 6.2^{\circ}$; dot density $=12.5 \mathrm{dots} / \mathrm{deg}^{2} ; \mathrm{CSF}=0.3 \mathrm{cpd}$. Scale at the top of the figure indicates the percepts evoked by the dot pattern at different ranges of angular velocities.

The association between the impairment of stereopsis and OKN was demonstrated in several ways. First, the appearance of comet-like tails appended to the moving dots indicated smooth eye-tracking movements. Second, a high degree of correlation between $\mathrm{OKN}$ periods and nonstereopsis periods was demonstrated by direct eyemovement measurements. Third, when smooth tracking eye movements or OKN were eliminated by short exposure durations or by prolonged to-and-fro motion, no equivalent impairment in stereopsis was evident. These results suggest that the dots' displacement per se does not affect stereopsis; rather, the effect is mediated by the OKN.

This analysis does not imply that OKN in itself is the main cause for the impairment. Several studies show that OKN and stereopsis can coexist. For example, Fox, Lehmkuhle, and Leguire (1978) were able to evoke and maintain $\mathrm{OKN}$ by cyclopean moving bars. Adler and Grüsser (1982) showed that stereopsis can be obtained during $\mathrm{OKN}$ and smooth pursuit. Also, in our experimental setup, when the stereograting moved with the dots, or when their orientation was horizontal rather than vertical, stereopsis was easily obtained at the same dot AVs that had previously impaired it (Vardi \& Hadani, in press). These findings suggest that the invisible monocular contours in one scope (A) did not cause the impairment, because they were present when the stereograting moved with the dots and were absent when the stereograting was horizontal. Moreover, $\mathrm{OKN}$ alone is not sufficient for the impairment; an additional factor is required. Inspection of all cases in which stereopsis was not affected during OKN or smooth pursuit, compared with the cases in which stereopsis was impaired, reveals an essential difference in stimulus configuration between the two cases: Stereop- 
A
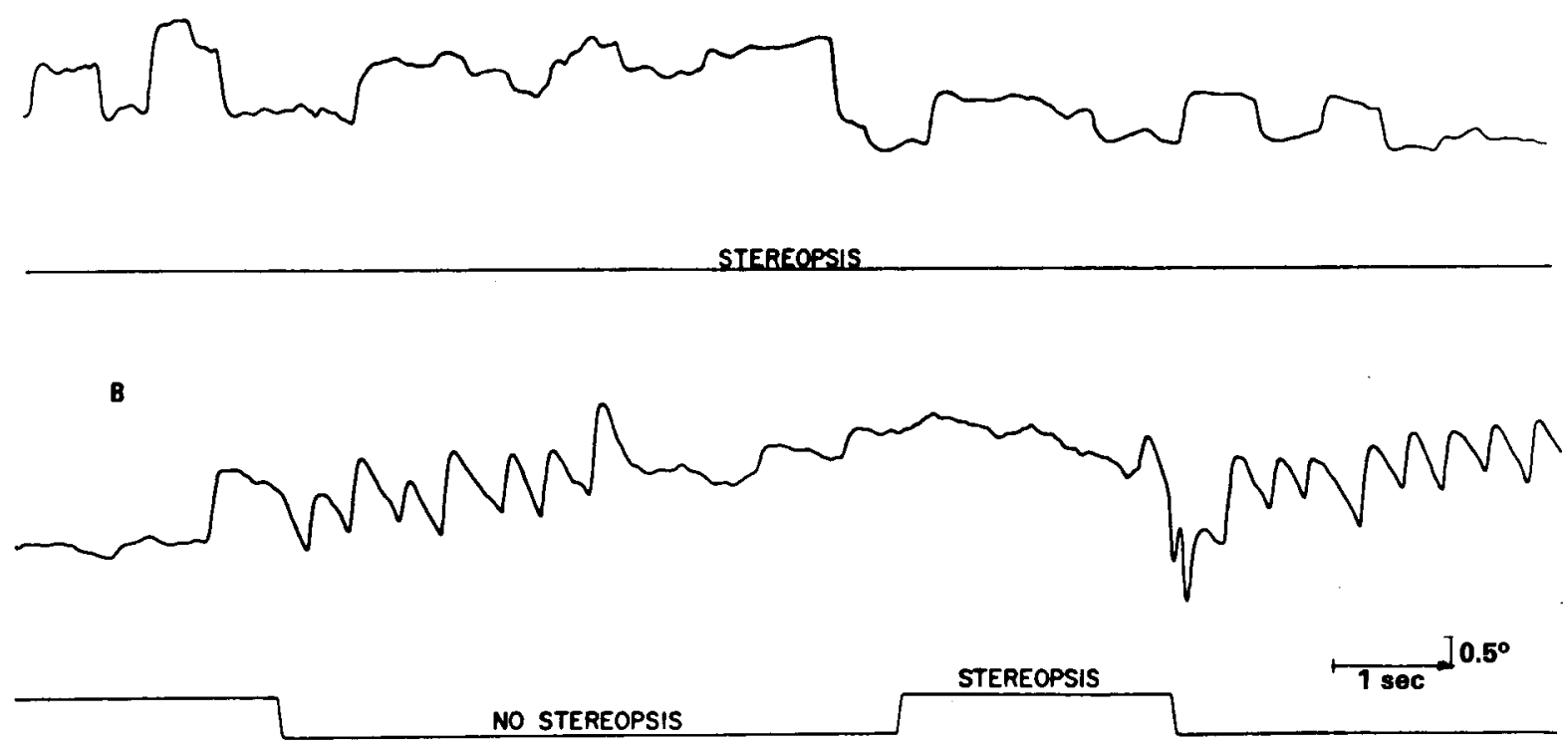

Figure 5. Typical eye-movements records for Subject N.V. at $0.1 \% \mathrm{sec}$ (A) and $4.4^{\circ} / \mathrm{sec}$ (B). The lower lines are records of the marker channel with which the subject signaled the occurrence of stereopsis.

sis was impaired during OKN only when the disparity magnitude of the tracked dots was changed along their movement path.

What can be the neural explanation for the impairment? Based on the experimental conditions for the stereopsis impairment phenomenon and known perceptual and neuronal mechanisms, we propose the following tentative explanation. Unidirectional movement of the dot field triggers OKN. In the smooth tracking phase of each OKN sweep, a given dot is locked (within tracking error) to a fixed retinal locus. Because of the retinotopic mapping of cells in areas 17, 18, and 19 (Poggio, 1980; these areas are henceforth referred to as the cyclopean retina), this dot would also be locked to a particular locus in the cyclopean retina. As this dot disparity changes (in the present case, due to its movement across the stereograting edges), so does the pattern of excitation of that locus. These changes in the pattern of excitation are similar to those obtained by pure temporal modulation of the input to that cortical element.

The above explanation can be expressed in different terms. Accurate tracking of the dots effectively nullifies

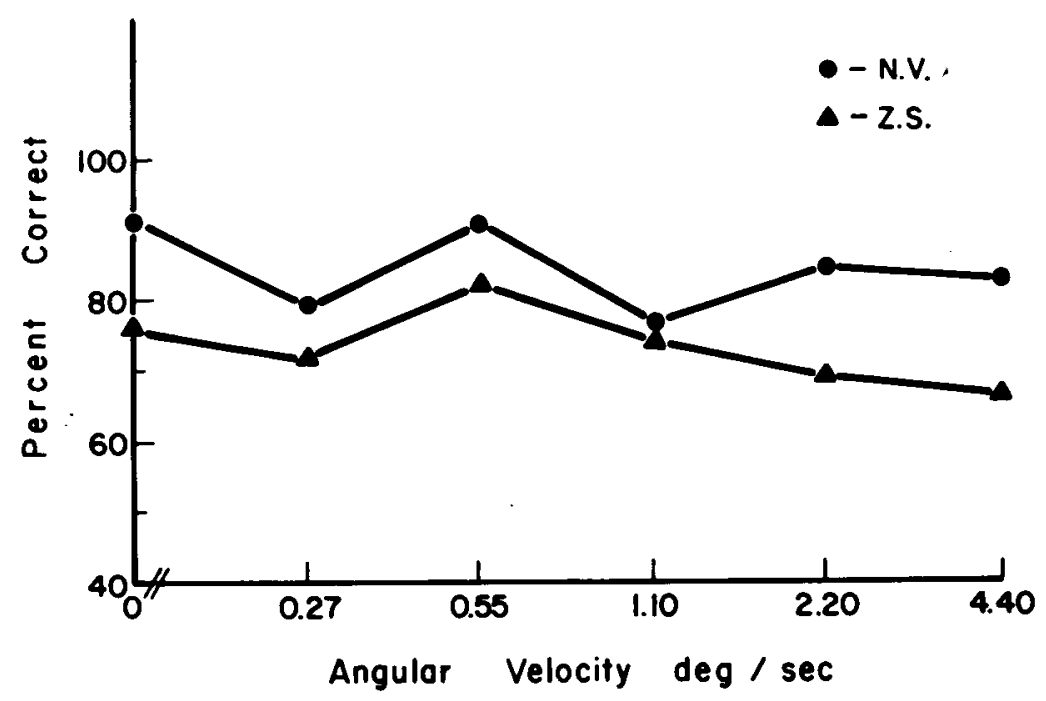

Figure 6. Percent correct response as a function of stimulus angular velocity for Subjects N.V. and Z.S. at 100-msec exposure duration. Cyclopean spatial frequency $=3.2 \mathrm{cpd}$; each data point is the average of at least 50 trials. 
their motion on the retinas and consequently on the cyclopean retina. Thus, the static stereograting can be regarded as moving (or sweeping) on the cyclopean retina in a direction opposite to the tracking. This "motion" creates temporal modulation of disparity information for a given disparity-sensitive cell. Whenever these temporal changes exceed a certain rate, disparity information is blurred and this results in reduced perceived depth. This suggestion is supported by studies on the temporal resolution limits of stereopsis during fixation. Disparity information is being temporally integrated during fixation over several hundred milliseconds. This was revealed by depth-reversal experiments in which the amplitude of motion in depth was reduced by increasing the rate of disparity modulation and was eliminated or changed into two simultaneous planes in depth at about $6 \mathrm{~Hz}$ (Neill, 1981; Norcia \& Tyler, 1984; Regan \& Beverly, 1973; Richards, 1972; White \& Odom, 1985). Above $6 \mathrm{~Hz}$, up to $14 \mathrm{~Hz}$, no motion in depth was seen, but two pulsating depth planes were seen simultaneously (Norcia \& Tyler, 1984). Thus, the 6-Hz limit indicates the lower limit of the simultaneity region in cyclopean motion. A similar temporal limit, of 5 to $6 \mathrm{~Hz}$, was found by Julesz and Payne (1968) for cyclopean rotational or lateral (apparent) movement. They reported that as the stimulation frequency was increased, the amplitude of interpolated to-and-fro movement was reduced, and at about $6 \mathrm{~Hz}$, a single cyclopean bar was seen standing still. Above $6 \mathrm{~Hz}$, the two cyclopean bars were seen simultaneously. According to the above explanation, such an integration process was also active in the present experiments during smooth tracking eye movements, and it averaged the disparity values of the dots.

Our explanation can also account for Adler and Grüsser's (1982) results. Their subjects smoothly tracked a stroboscopically illuminated vertical static stereograting. Stereopsis was obtained only when the eyes' velocity equaled the product of the strobe frequency and the stereograting period. Stereoscopic depth was greatly reduced or eliminated otherwise. Therefore, in Adler and Grüsser's paradigm, stereopsis was obtained only when the stereograting was retinally displaced from one strobe pulse to the next by a complete period or by integral period multiples, that is, when disparity value per given location on the cyclopean retina was not considerably changed.

Considering the types of eye movements induced by the display and the temporal limit of the disparity integration process, we can explain why the impairment in our study only showed up at moderate AVs $\left(1^{\circ}-5.5^{\circ} / \mathrm{sec}\right)$. For AVs lower than $1 \% / \mathrm{sec}$, the temporal modulation rate was low and did not affect stereopsis at suprathreshold disparities (indeed, an effect was revealed in the stereoacuity experiment, as shown in Figure 3). For AVs higher than $5.5^{\circ} / \mathrm{sec}$, the eyes switched to a fixation mode, as was indicated by the appearance of the alpha stripes. In this mode the disparity magnitude per specific retinal area stays roughly constant. Our explanation predicts that increasing the CSF should increase stereopsis impairment because of its effect on the rate of disparity modulation. The effect of CSF, however, was weak, presumably because of the restricted range of CSFs that we could utilize. ${ }^{1}$ We are currently investigating the effect of higher CSFs on impairment, using other psychophysical methods. The results of these investigations clearly support our explanation. They show, first, that the impairment in stereopsis depends on the product of CSF and AV (which means the temporal modulations of disparity) and not on AV; and second, that the stereoscopic depth is inversely proportional to the product of CSF and AV in the range of 3 to $7 \mathrm{~Hz}$ (Vardi \& Hadani, 1986, in press).

Finally, the transition between the alpha-stripes and comet-tail percepts is expressed by a drastic change in the perceptual quality of the monocular elements. Although these effects were not the main concern of the present study and served only as a probe for the observers' eye-movement behavior, it is interesting to know how effective these monocular features are as an input to the stereoscopic mechanism. This question could not be adequately addressed in the present display because both oscilloscope CRTs had the same persistence. Therefore, if, for example, a given dot disparity was changed from zero to a crossed disparity value, its tail disparity was changed as well, and the tailed dot as a whole appeared closer to the observer even if the tail was extended so that it crossed a stereoscopic edge. In the future we intend to get into this question by utilizing two CRTs, one having a very low and the other a very high persistence. In this case the dots will remain a binocular input but the tails will be a monocular input. The perceived depth level of the tails, in particular those that cross the stereoscopic edges, may indicate how effective this stimulus is for stereopsis. Thus more insight into the relationships between monocular feature extraction and stereoscopic vision would be gained.

\section{REFERENCES}

ADLER, B., Bock, O., \& Grüsser, O. J. (1981). Alpha-stripes: A spatial periodicity appearing in stroboscopically illuminated moving random dot patterns. Vision Research, 21, 913-924.

ADLER, B., \& GRüsSER, O. J. (1979). Apparent movement and appearance of periodic stripes during eye movements across a stroboscopically illuminated random dot pattern. Experimental Brain Research, 32, 537-550.

ADLER, B.,. \& GRÜSSER, O. J. (1982). Sigma-movement and optokinetic nystagmus elicited by stroboscopically illuminated stereopatterns. Experimental Brain Research, 47, 353-364.

Farrell, J. E., Putnam, T., Shepard, R. N. (1984). Pursuit-locked apparent motion. Bulletin of the Psychonomic Society, 22, 345-348.

FoX, R., LehmKuHLE, S., \& LeguiRe, L. E. (1978). Stereoscopic contours induce optokinetic nystagmus. Vision Research, 18, 1189-1192.

GiBson, J. J. (1966). The senses considered as perceptual systems. Boston: Houghton Mifflin.

HADANI, I. (1984a). Apparent motion perception from dynamic random dot patterns by dot correspondence in non-contiguous frames. Investigative Ophthalmology \& Visual Science, 3(Suppl. 25), 13.

HaDaNi, I. (1984b). Visual comet tails in unidirectional apparent motion of random-dot pattern. Proceedings of the 7th Annual Meeting of the ECVP. Perception, 13, A40. (Abstract) 
Julesz, B. (1971). Foundations of cyclopean perception. Chicago: University of Chicago Press.

Julesz, B., \& PAYNE, R. A. (1968). Differences between monocular and binocular stroboscopic movement perception. Vision Research, 8, 433-444.

Koenderink, J. J., \& VAN Doorn, A. J. (1980). Dual percept of movement and spatial periodicity in stroboscopically illuminated moving noise patterns. Journal of the Optical Society of America, 70, 456-458.

LEE, D. N. (1970). Binocular stereopsis without spatial disparity. Perception \& Psychophysics, 9(2B), 216-218.

MoRGAN, M. J. (1981). How pursuit eye movements can convert temporal into spatial information. In D. F. Fisher, R. A. Monty, \& J. W. Senders (Eds.), Eye novements: Cognition and visual perception (pp. 111-133). Hillsdale, NJ: Erlbaum.

Nakayama, K., Silverman, G. H., Macleod, D. I. A., \& MulliGAN, J. (1985). Sensitivity to shearing and compressive motion in random dots. Perception, 14, 225-238.

NeILL, R. A. (1981). Spatio-temporal averaging and the dynamic visual noise stereophenomenon. Vision Research, 21, 673-682.

Norcia, A. M., TYLER, C. W. (1984). Temporal frequency limits for stereoscopic apparent motion processes. Vision Research, 24, 395-401.

Pogcio, G. F. (1980). Central neural mechanism in vision. In V. R. Mountcastle (Ed.), Medical physiology (14th ed., Vol. 1). St. Louis: Mosby.

REgan, D., \& BeVERLy, K. I. (1973). Some dynamic features of depth perception. Vision Research, 13, 2369-2379.
RichaRDS, W. (1972). Response functions for sine-and-square-wave modulations of disparity. Journal of the Optical Society of America, 62, 907-911.

VARDI, N., \& HADANI, I. (1986). Spatiotemporal integration in static disparity field composed of random dots in coherent motion. Proceedings of the 9th Annual Meeting of the ECVP. Perception, 15, 1, A40b. (Abstract)

VARDI, N., \& HADANI, I. (in press). Stereopsis impairment during smooth pursuit eye tracking. Brain Behavior \& Evolution.

WESTHEIMER, G. (1954). Eye movements responses to a horizontally moving visual stimulus. Archives of Ophthalmology, 52, 932-941.

Westhejmer, G., McKeE, S. P. (1978). Stereoscopic acuity for moving retinal images. Journal of the Optical Society of America, 68, $450-455$.

WhITE, K. D., \& ODOM, J. V. (1985). Temporal integration in global stereopsis. Perception \& Psychophysics, 37, 139-144.

\section{NOTE}

1. The reason for not utilizing higher CSFs in this study was pragmatic rather than technical. Our psychophysical method was based on resolving the position of the odd stripes relative to the even. This could be reliably done only up to $4.4 \mathrm{cpd}$.

(Manuscript received July 21, 1986; revision accepted for publication March 23, 1987.) 